\title{
ANÁLISE DA EFETIVIDADE DA TERAPÊU- TICA NUTRICIONAL NO CONTROLO DA DIABETES MELLITUS TIPO 2
}

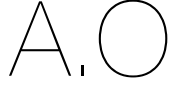

ARTIGO ORIGINAL

1 Unidade de Recursos Assistenciais Partilhados do ACeS Porto Ocidental, Administração Regional de Saúde do Norte- IP Rua Vila Nova, s/n, 4100-503 Porto, Portugal

*Endereço para correspondência:

sabel Monteiro

URAP-polo de Aldoar, ACeS

Porto Ocidental,

Pua Vila Nova, s/n,

4100-503 Porto, Portuga

impribeiro@arsnorte.min-saude.pt

Histórico do artigo

Recebido a 29 de fevereiro de 2020 Aceite a 27 de março de 2021

\section{NUTRITIONAL THERAPY EFFECTIVENESS ANALYSIS IN THE CONTROL OF TYPE 2 DIABETES MELLITUS}

Clara Novais'; Isabel Monteiro' ${ }^{*}$

RESUMO

INTRODUÇÃo: A Terapêutica Nutricional individualizada constitui, a par da farmacoterapia, um fator fundamental no controlo da Diabetes Mellitus tipo 2. A avaliação da efetividade da Terapêutica Nutricional em adultos com Diabetes Mellitus tipo 2 passa pelo controlo glicémico, redução de fatores de risco cardiovascular, redução do peso e/ou prevenção do seu ganho, diminuição do uso de fármacos e aumento da qualidade de vida.

OBJETIVOS: Este estudo teve como objetivo analisar a efetividade da Terapêutica Nutricional no controlo da Diabetes Mellitus tipo 2 em adultos pela avaliação da evolução do perfil glicémico e evolução ponderal

METODOLOGIA: Recolheram-se dados sociodemográficos, analíticos e antropométricos de 75 indivíduos com diagnóstico de Diabetes Mellitus tipo 2, acompanhados durante 3 anos numa Consulta de Nutrição dos Cuidados de Saúde Primários. Os resultados são apresentados como média \pm desvio-padrão. Para o estudo estatístico aplicou-se o teste t-Student para variáveis normais, e o teste Wilcoxon para as restantes variáveis.

RESULTADOS: Observaram-se melhorias, estatisticamente significativas na avaliação antropométrica: peso (-0,8 Kg) e ĺndice de Massa Corporal $\left(-0,7 \mathrm{Kg} / \mathrm{m}^{2}\right)$ e perfil glicémico: HbA1c (-0,6\%) e glicemia em jejum (-21 mg/dl) após 3 meses de Terapêutica Nutricional, bem como a longo prazo: peso (-7,8 Kg), Índice de Massa Corporal (-2,5 Kg/m²) e glicemia em jejum(-28 mg/dl).

CONCLUSõEs: Perante as melhorias significativas verificadas ao nível da evolução ponderal e perfil glicémico 3 meses após o início da Terapêutica Nutricional e quando se comparou os dados iniciais com os obtidos aos 3 anos de Terapêutica Nutricional, é possível inferir que a Terapêutica Nutricional individualizada é efetiva no controlo da Diabetes Mellitus tipo 2, pelo que deveria ser disponibilizada a todos os indivíduos com este diagnóstico. Assim, é importante sensibilizar a população para a sua relevância e importância da sua manutenção a longo prazo e os gestores para a dotação de rácios adequados de Nutricionistas nos Cuidados de Saúde Primários.

\section{PALAVRAS-CHAVE}

Diabetes, Perfil glicémico, Peso, Terapêutica nutricional

\section{ABSTRACT}

INTRODUCTION: Individualized Nutritional Therapy is, together with pharmacotherapy, a fundamental factor in the control of type 2 Diabetes Mellitus. Evaluation of the effectiveness of Nutritional Therapy in adults with type 2 Diabetes Mellitus involves glycemic control, reduction of cardiovascular risk factors, weight reduction and/or prevention of weight gain, decreased drug use and increased quality of life.

OBJECTIVES: This study aimed to analyze the effectiveness of Nutritional Therapy in controlling type 2 Diabetes Mellitus in adults by assessing the evolution of glycemic profile and weight evolution.

METHODOLOGY: Sociodemographic, analytical and anthropometric data were collected from 75 subjects with type 2 Diabetes Mellitus followed for 3 years at the Nutrition Consultation of a Primary Health Care Unit. Results are presented as mean \pm standard deviation. For the statistical study we used the Student t-test for normal variables and the Wilcoxon test for the remaining variables. RESULTS: Statistically significant improvements were observed in anthropometric assessement: weight (-0.8 kg) and Body Mass Index $\left(-0.7 \mathrm{~kg} / \mathrm{m}^{2}\right)$ and glycemic profile: HbA1c $(-0.6 \%)$ and fasting blood glucose $(-21 \mathrm{mg} / \mathrm{dl})$ after 3 months of Nutritional Therapy, as well as long-term: weight $(-7.8 \mathrm{~kg})$, Body Mass Index $\left(-2.5 \mathrm{~kg} / \mathrm{m}^{2}\right)$ and fasting blood glucose $(-28 \mathrm{mg} / \mathrm{dll})$.

CONCLUSIONS: Considering the significant improvements were observed in terms of weight evolution and glycemic profile 3 months after the onset of Nutritional Therapy and when the initial data were compared with those obtained at 3 years of Nutritional Therapy, it can be inferred that individualized Nutritional Therapy is effective in controlling type 2 Diabetes Mellitus, therefore, it should be available to all individuals with this diagnosis. Thus, it is important to make the population aware of its relevance and importance of its long-term maintenance, and managers to provide adequate ratios of Nutritionists in Primary Health Care.

KEYWORDS

Diabetes, Glycemic profile, Weight, Nutritional therapy 


\section{INTRODUÇÃO}

A Diabetes Mellitus (DM) é uma doença metabólica, cuja prevalência mundial, em adultos, quase duplicou de 1980 a 2014, passando de $4,7 \%$ para $8,5 \%$ e perspetiva-se que nos próximos 20 anos atinja mais de $20 \%$ da população (1). Segundo a Organização para a Cooperação e Desenvolvimento Económico (OCDE), Portugal é um dos países com a taxa de prevalência mais alta da Europa (2). De acordo com os dados publicados em 2017 no Relatório Anual do Observatório Nacional da Diabetes, Portugal surge com uma prevalência de 13,3\% da população em 2015 (valor correspondente a mais de um milhão de portugueses), dos quais se estima que $44 \%$ desconheça ter a doença (3). Trata-se de uma doença crónica e progressiva que pode trazer graves repercussões para a saúde e bem-estar individual, estando ainda associada a elevados custos para o Serviço Nacional de Saúde, sobretudo no que concerne ao aumento contínuo do consumo de fármacos. Atualmente, o tratamento da DM tipo 2 e das suas complicações representam cerca de $10 \%$ das despesas em saúde, o que corresponde a 1\% do PIB Nacional. Neste sentido, a prevenção e o seu diagnóstico, bem como o diagnóstico precoce das suas complicações e respetivo tratamento, são prioridades para reverter o cenário atual (4).

De acordo com as recomendações da American Diabetes Association (ADA) (4), a par da terapêutica farmacológica, a Terapêutica Nutricional individualizada (TN) revela-se essencial no controlo da diabetes, tendo como principais objetivos otimizar o estado nutricional, e consequentemente a saúde, adquirir e preservar bom controlo metabólico e assim evitar ou retardar as complicações associadas. Diversos estudos (5) têm demonstrado fortes evidências que suportam a eficácia da TN, nomeadamente na melhoria dos níveis glicémicos, na redução do peso e consequente diminuição da resistência à insulina. Os resultados obtidos num estudo (6), realizado em indivíduos obesos com DM tipo 2, mostraram que o grau de restrição energética, melhora o controlo glicémico, bem como a sensibilidade à insulina. Assim, a evidencia atual (4) considera essencial providenciar uma TN estabelecida de forma personalizada, considerando o estado de saúde e estado nutricional, o perfil metabólico (perfil lipídico, função renal, etc.), assim como o padrão de consumo, aspetos culturais e escolhas/ preferências alimentares, garantindo o aporte energético apropriado ao estabelecimento e/ou manutenção de um peso saudável. A TN individualizada constitui um importante pilar no controlo da DM tipo 2, no entanto exige monitorização, e reforço constante para a sua manutenção. Segundo a Academy of Nutrition and Dietetics (7) diferentes intervenções educacionais dirigidas a diabéticos associam-se a menores taxas de hospitalização e menores custos com saúde. Quando estas intervenções eram dinamizadas por nutricionistas verificou-se maior redução de hospitalizações e redução substancial nas despesas hospitalares. Os autores referem que tais resultados sugerem que a prestação desses serviços no contexto dos Cuidados de Saúde Primários (CSP) pode ser altamente rentável para o Sistema de Saúde (7).

\section{OBJETIVOS}

Foi objetivo deste trabalho avaliar a efetividade da TN individualizada no controlo glicémico, pela análise da evolução do perfil glicémico [hemoglobina glicada ( $\mathrm{HbA1c}$ ) e níveis glicémicos em jejum] e de dados antropométricos (redução do peso e Índice de Massa Corporal (IMC), quando em excesso) de indivíduos adultos com DM tipo 2, acompanhados em Consulta de Nutrição nos CSP durante 3 anos.

\section{METODOLOGIA}

Para a concretização do objetivo proposto, foi desenhado o presente estudo observacional longitudinal, de carácter retrospetivo, tendo sido analisados processos clínicos de utentes da consulta de Nutrição da Unidade de Recursos Assistenciais Partilhados (URAP) - polo de Aldoar do Agrupamento de Centros de Saúde (ACeS) Porto Ocidental, após solicitação de parecer ao Conselho Clínico e da Saúde da Instituição e à Comissão de Ética da Administração Regional de Saúde do Norte.

\section{Participantes}

Foram elegíveis para o estudo, indivíduos com DM tipo 2 referenciados à Consulta de Nutrição. Assim, avaliaram-se 110 processos clínicos de indivíduos adultos (idade superior a 18 anos), tendo-se excluído os que foram a uma só Consulta de Nutrição bem como os que não apresentavam pelo menos duas avaliações de HbA1c ou glicemia em jejum no período em análise. Desta forma, obteve-se uma amostra final de 75 participantes.

\section{Recolha de Informação}

A recolha de dados foi efetuada mediante a consulta dos processos clínicos dos utentes elegíveis. Assim, recolheram-se dados obtidos na primeira consulta: sociodemográficos, dados antropométricos (peso, altura), informação relativa a antecedentes de doença (hipertensão, obesidade ou dislipidemia) e respetiva medicação e foram ainda recolhidos dados bioquímicos ( $\mathrm{HbA} 1 \mathrm{c}$ e glicemia em jejum) registados na primeira consulta. Após 3, 6 e 9 meses e também após 1, 2 e 3 anos, os dados bioquímicos e o peso também foram recolhidos. Calculou-se o IMC, pela fórmula de Quetelet, e categorizou-se de acordo com a classificação da Organização Mundial da Saúde (8).

\section{Análise Estatística}

Procedeu-se à análise estatística, recorrendo ao software IBM Statistics, Statistical Package for the Social Sciences (SPSS) versão 25. As variáveis categóricas foram descritas através das suas frequências absolutas (n) e frequências relativas (\%). As variáveis contínuas e discretas foram descritas através da média \pm desvio-padrão, após verificação da normalidade da distribuição, mediante o teste de Kolmogorov-Smirnov ( $n>50)$. Para a comparação de médias, em amostras emparelhadas e variáveis com distribuição normal, aplicouse o teste t-Student. Para a comparação de médias, em amostras emparelhadas e variáveis com distribuição não normal, aplicou-se o teste de Wilcoxon. Foi considerado um nível de significância de 5\%.

\section{RESULTADOS}

\section{Caracterização da Amostra}

Dos 75 indivíduos com DM tipo 2 elegíveis para o estudo, 46 eram do sexo masculino, ou seja, $61,3 \%$ dos participantes. A idade média foi de $61( \pm 10,0)$ anos, sendo a faixa etária entre 60-69 anos a mais prevalente (seguindo-se a faixa entre os 50-59 anos), não se verificando diferenças significativas na média de idades entre sexos $(p=0,913)$. Cerca de $52(69,3 \%)$ indivíduos apresentavam HTA e 56 $(74,7 \%)$ apresentavam dislipidemia, $43(57,3 \%)$ apresentavam ambas e somente 10 dos indivíduos não apresentavam nenhum destes fatores de risco. Dos participantes que apresentavam dislipidemia, 28 (50\%) tomavam medicação para controlo do perfil lipídico e do total de utentes hipertensos, observou-se que 42 efetuavam a respetiva medicação (80,8\%). De forma a caracterizar melhor a amostra, foi ainda averiguada a frequência de abandono à TN no período em estudo, constatando-se, a ausência de abandono nos primeiros 3 meses de TN. No entanto, relativamente ao n. ${ }^{\circ}$ total de abandonos, verifica-se que $22 \%$ abandona definitivamente a TN ao fim de 6 meses, $28 \%$ após 9 meses, $15 \%$ abandonou a consulta no final de 1 ano de 
seguimento, 22\% aos 2 anos e 11\% não compareceram à consulta no final do período em análise ( 3 anos). Estes dados revelam um elevado absentismo à Consulta de Nutrição, pelo que se verificaram missings de informação que, por sua vez, condicionaram os resultados.

\section{Estado Nutricional}

Relativamente ao estado nutricional (Tabela 1), o peso médio dos indivíduos na primeira consulta foi de $85,7( \pm 15,8) \mathrm{kg}$, o qual foi diminuindo ao longo do tempo, verificando-se na última avaliação registada (após 3 anos) uma redução de 7,8 kg $(85,7( \pm 15,8) \mathrm{kg}$ vs. $77,9( \pm 13,4) \mathrm{kg}$ ) e uma perda ponderal de $4,1 \%$ face ao peso inicial). Os homens perderam 4,3\% do seu peso inicial (de 88,1 para 79,6 $\mathrm{kg}$ ), enquanto as mulheres perderam cerca de 4,2\% (de 81,2 para $76,5 \mathrm{~kg})$. Constatou-se que o IMC médio inicial era $32,0( \pm 5,7) \mathrm{Kg} / \mathrm{m}^{2}$ (obesidade grau 1), e que este diminuiu progressivamente atingindo $29,5( \pm 4,9) \mathrm{Kg} / \mathrm{m}^{2}$ (pré-obesidade) ao fim dos 3 anos. A redução do IMC médio foi mais marcada nos homens (30,6 para $\left.27,3 \mathrm{~kg} / \mathrm{m}^{2}\right)$ do que nas mulheres (de 34,1 para $31,3 \mathrm{~kg} / \mathrm{m}^{2}$ ).

Para averiguar se a TN individualizada permite melhorar o estado nutricional, comparou-se o peso e IMC médio reavaliados em cada uma das consultas subsequentes (aos 3, 6, 9 meses, 1, 2 e 3 anos) com os dados da consulta anterior (Tabela 1). Constataram-se melhorias significativas no peso após 3 meses de seguimento em Consulta de Nutrição $(85,7 \pm 15,8 \mathrm{~kg}$ vs. $84,9 \pm 15,0 \mathrm{~kg}, \mathrm{p}<0,001)$ e entre $02 .^{\circ}$ e o 3. ${ }^{\circ}$ ano de seguimento $(80,9 \pm 14,2 \mathrm{~kg}$ vs. $77,9 \pm 13,4 \mathrm{~kg}, \mathrm{p}=0,008)$. Os resultados obtidos ao nível do peso foram, consequentemente, refletidos no IMC dos utentes, pelo que se constatou igualmente melhorias significativas após 3 meses, da primeira consulta (32,0 $\mathrm{kg} / \mathrm{m}^{2}$ vs. $\left.31,6 \mathrm{~kg} / \mathrm{m}^{2}, \mathrm{p}<0,001\right)$ bem como entre o $2 .^{\circ}$ e $3 .^{\circ}$ ano de seguimento $\left(29,8 \mathrm{~kg} / \mathrm{m}^{2}\right.$ vs. $\left.29,5 \mathrm{~kg} / \mathrm{m}^{2}, \mathrm{p}=0,019\right)$. Foi também apurada a existência de diferenças significativas na redução do peso e, consequentemente, do IMC, quando se compararam os resultados obtidos na primeira consulta com os dados apurados após 3 anos de $\mathrm{TN}\left(85,7 \mathrm{~kg} \pm 15,8 \mathrm{~kg}\right.$ vs. $77,9 \mathrm{~kg}( \pm 13,4), \mathrm{p}=0,01$ e $32,0 \pm 4,6 \mathrm{~kg} / \mathrm{m}^{2}$ vs. $\left.29,5 \pm 4,9 \mathrm{~kg} / \mathrm{m}^{2}, \mathrm{p}=0,013\right)$.

\section{Perfil Glicémico}

Quanto ao perfil glicémico dos participantes, verificou-se descida do valor médio da $\mathrm{HbA1c}$, que inicialmente era $7,7( \pm 2,1) \% \%$ e após 3 anos passou para $7,2( \pm 1,4) \%$, não obstante, verificou-se após 6 meses de TN que a média atingiu o valor mais baixo de todo o estudo $(7,0( \pm 1,4) \%)$. No caso da glicemia em jejum, o valor médio inicial foi 163( \pm 63$) \mathrm{mg} / \mathrm{dL}$, tendo diminuído e permanecido inferior a este valor no decurso do estudo e embora se observe um ligeiro aumento aos 9 meses e 1 ano, atingiu-se o valor médio mais baixo de ao fim de 2 anos $(128 \pm 34 \mathrm{mg} / \mathrm{dL})$. Aos 3 anos de acompanhamento de TN encontrou-se uma glicemia média de $135( \pm 45) \mathrm{mg} / \mathrm{dL}$. Para avaliar se a TN individualizada permite melhorar o controlo glicémico, foram comparadas as médias de $\mathrm{HbA} 1 \mathrm{c}$ e da glicemia em jejum reavaliados em cada uma das consultas subsequentes (aos 3, 6, 9 meses, 1, 2 e 3 anos) com os dados da consulta anterior, bem como a evolução entre a primeira consulta e os dados do final do estudo. Assim, verificou-se (Tabela 2) uma redução significativa para a HbA1c ao fim de 3 meses $(p=0,004)$ de acompanhamento em consulta $(7,7( \pm 2,1) \%$ vs. $7,1( \pm 1,1) \%)$ e no caso da glicemia em jejum, foram igualmente observadas melhorias significativas ( $p=0,001)$ ao fim de 3 meses (163 $\mathrm{mg} / \mathrm{dL}$ vs. $142 \mathrm{mg} / \mathrm{dL}$ ), bem como quando comparado o valor no início da TN com o valor obtido ao fim dos 3 anos (163mg/dL vs. $135 \mathrm{mg} /$ $d L)$ de $T N(p=0,025)$.

\section{DISCUSSÃO DOS RESULTADOS}

Segundo a ADA e a European Association for the Study of Diabetes $(9,10)$ a gestão da diabetes deve ser centrada no doente e combinar modificação do estilo de vida com terapia farmacológica. A ADA

\section{Tabela 1}

Parâmetros antropométricos obtidos na primeira consulta e após 3, 6, 9 meses e 1, 2 e 3 anos de seguimento em Consulta de Nutrição

\begin{tabular}{|c|c|c|c|c|c|c|c|}
\hline PARÂMETROS & $1^{\mathrm{a}}$ & 3 MESES & 6 MESES & 9 MESES & 1 ANO & 2 ANOS & 3 ANOS \\
\hline $\mathrm{N}$ & 75 & 74 & 56 & 38 & 40 & 28 & 22 \\
\hline $\begin{array}{l}\text { Peso } \mathbf{( k g )} \\
\text { Média e desvio-padrão }\end{array}$ & $\begin{array}{c}85,7 \\
( \pm 15,8)\end{array}$ & $\begin{array}{c}84,9^{\star} \\
( \pm 15,0)\end{array}$ & $\begin{array}{c}83,7 \\
( \pm 15,5)\end{array}$ & $\begin{array}{c}84,2 \\
( \pm 15,5)\end{array}$ & $\begin{array}{c}82,1 \\
( \pm 15,3)\end{array}$ & $\begin{array}{c}80,9 \\
( \pm 14,2)\end{array}$ & $\begin{array}{l}77,9^{\star} \bullet \\
( \pm 13,4)\end{array}$ \\
\hline $\mathrm{N}$ & 75 & 74 & 56 & 38 & 40 & 38 & 22 \\
\hline $\begin{array}{l}\text { IMC }\left(\mathbf{k g} / \mathbf{m}^{2}\right) \\
\text { Média e desvio-padrão }\end{array}$ & $\begin{array}{l}32,0 \\
( \pm 5,7)\end{array}$ & $\begin{array}{l}31,6^{\star} \\
( \pm 5,4)\end{array}$ & $\begin{array}{l}31,3^{*} \\
( \pm 5,3)\end{array}$ & $\begin{array}{c}31,2 \\
( \pm 5,5)\end{array}$ & $\begin{array}{c}30,7 \\
( \pm 5,5)\end{array}$ & $\begin{array}{c}29,8 \\
( \pm 4,6)\end{array}$ & $\begin{array}{l}29,5^{\star} \bullet \\
( \pm 4,9)\end{array}$ \\
\hline
\end{tabular}

* melhorias significativas $(p<0,05)$ face à consulta anterior

- melhorias significativas $(p<0,05)$ entre a primeira avaliação e ao fim de 3 anos de TN

IMC: Índice de Massa Corporal

\section{Tabela 2}

Hemoglobina glicada e Glicemia em jejum registadas na primeira consulta e após 3, 6, 9 meses e 1, 2 e 3 anos de seguimento em Consulta de Nutrição

\begin{tabular}{|c|c|c|c|c|c|c|c|}
\hline PARÂMETROS & $1^{a}$ & 3 MESES & 6 MESES & 9 MESES & 1 ANO & 2 ANOS & 3 ANOS \\
\hline N & 66 & 39 & 35 & 27 & 53 & 41 & 38 \\
\hline $\begin{array}{l}\text { HbA1c (\%) } \\
\text { Média e desvio-padrão }\end{array}$ & $\begin{array}{c}7,7 \\
( \pm 2,1)\end{array}$ & $\begin{array}{c}7,1^{\star} \\
( \pm 1,1)\end{array}$ & $\begin{array}{c}7,0 \\
( \pm 1,4)\end{array}$ & $\begin{array}{c}7,3 \\
( \pm 1,4)\end{array}$ & $\begin{array}{c}7,1 \\
( \pm 1,4)\end{array}$ & $\begin{array}{c}7,2 \\
( \pm 1,2)\end{array}$ & $\begin{array}{c}7,2 \\
( \pm 1,4)\end{array}$ \\
\hline $\mathrm{N}$ & 66 & 34 & 22 & 20 & 26 & 26 & 27 \\
\hline $\begin{array}{l}\text { Glicemia em jejum } \\
\text { Média e desvio-padrão }\end{array}$ & $\begin{array}{c}163 \\
( \pm 63)\end{array}$ & $\begin{array}{l}142^{*} \\
( \pm 53)\end{array}$ & $\begin{array}{c}138 \\
( \pm 42)\end{array}$ & $\begin{array}{c}142 \\
( \pm 43)\end{array}$ & $\begin{array}{l}142 \\
( \pm 57)\end{array}$ & $\begin{array}{c}128 \\
( \pm 34)\end{array}$ & $\begin{array}{l}135 \bullet \\
( \pm 45)\end{array}$ \\
\hline
\end{tabular}

* melhorias significativas $(p<0,05)$ face à consulta anterior

- melhorias significativas $(p<0,05)$ entre a primeira avaliação e ao fim de 3 anos de TN 
recomenda ainda que os indivíduos com sobrecarga ponderal reduzam $5 \%$ ou mais o seu peso (10). A restrição energética, e consequente perda de peso, assume especial relevância no controlo desta doença, uma vez que, permite melhorar o estado nutricional, bem como o controlo glicémico e a sensibilidade à insulina. No estudo retrospetivo apresentado, a recolha e manutenção de dados é perturbada, sobretudo clínicos o que leva a que a efetividade da TN não se verifique ao longo de todo o período avaliado. Os participantes foram encaminhados para esta consulta por outro profissional de saúde, pelo que nem todos estariam disponíveis para a mudança. A falta de literacia também poderá ser uma condicionante no reconhecimento da relevância da alimentação no controlo da doença. O intervalo de tempo entre consultas, condicionado pela escassez de recurso humano (1 nutricionista/100000 utentes, em vez de 1/20000 como recomendado) será outro fator importante. Segundo o Diabetes Prevention Program (DPP) (11) nos primeiros 24 meses devem existir 16 consultas promotoras de redução da ingestão energética, de aumento de atividade física, capacitação para a automonitorização, para a manutenção de comportamentos de estilo de vida saudáveis, e para trabalhar desafios sociais e motivacionais. No entanto, apesar da diminuição do $\mathrm{n}$ de utentes (mais acentuada aos 6 meses) e, por conseguinte, missings de informação, no presente estudo, o impacto da TN é evidenciado pela diminuição progressiva do peso médio dos participantes ao longo do tempo, o qual diminuiu significativamente, verificando-se aos 3 anos uma perda média de $7,8 \mathrm{Kg}$, ou seja, uma redução de $4,1 \%$ do peso inicial, semelhante nos homens e nas mulheres (4,3\% vs. 4,2\%), não atingindo a perda de peso recomendada pela CDC (Centers for Disease Control and Prevention) de 5 a 7\% (12). Resultados de uma revisão sistemática (5) mostraram em 10 estudos que a TN se associou a perda de peso significativa, que oscilou entre 2,4 e 6,2 Kg, mas em 6 estudos não se encontrou variação de peso significativa. A melhoria do peso médio dos participantes foi, naturalmente, refletida no IMC, passando de um IMC médio de $32,0 \mathrm{Kg} / \mathrm{m}^{2}$ para um IMC final de $29,5 \mathrm{Kg} / \mathrm{m}^{2}$, reduzindo francamente o risco de morbilidade e mortalidade. Resultados da referida revisão sistemática (5) mostraram redução de IMC entre 0,3 e 2,1 em 9 estudos, enquanto em 6 deles não encontraram variação no IMC. No presente trabalho, aos 3 meses, foi encontrada uma redução significativa de 0,8 $\mathrm{Kg}$ no peso e de $0,4 \mathrm{Kg} / \mathrm{m}^{2}$ no IMC, valores um pouco inferiores aos encontrados por Coppell KJ, et al (13), que observou, igualmente, após a primeira consulta (aos 6 meses), uma perda de $2,1 \mathrm{Kg}$ e redução de $0,8 \mathrm{Kg} / \mathrm{m}^{2}$ no IMC. Neste estudo detetou-se ainda uma redução com significado estatístico entre $02 .^{\circ}$ e 3. ${ }^{\circ}$ ano ao nível do peso e IMC, bem como uma redução significativa entre os valores iniciais de peso e IMC e após os 3 anos de TN (Tabela 1). Algumas investigações mostram que a TN fornecida a curto prazo (nos primeiros 6 meses), pode reduzir o peso corporal em 10\% e manter a perda de peso por mais de um ano (14), pelo que os resultados obtidos no final do estudo demonstram a importância da manutenção a longo prazo da TN individualizada.

No que diz respeito ao controlo glicémico, resultados de vários estudos mostram que a TN conduz a reduções da HbA1c semelhantes ou superiores às obtidas com a farmacoterapia (5). Há evidência robusta para a eficácia obtida com TN fornecida por Nutricionistas, com reduções absolutas da HbA1c até $2,0 \%$ (na diabetes tipo 2) num período de 3 a 6 meses (5). O suporte contínuo da TN é útil para manter essas melhorias glicémicas (5). A variação nesta redução é justificada, por alguns autores, pelo tipo e duração da diabetes (5). Um estudo (15) mostrou reduções que variaram entre 0,25\% e 2,9\% na $\mathrm{HbA} 1 \mathrm{c}$, aos 3 e aos 6 meses de TN. No presente estudo, encontrou- -se redução respetivamente de 0,6\% e 0,7\% na HbA1c, embora não ajustado para o tempo de diagnóstico da doença.

Franz, MJ. et al (16), salientam a relevância da TN ser individualizada e referem que a duração desta se associa de forma direta com a redução do peso, mas não com o controlo glicémico, o que poderá ser explicado pela própria evolução da patologia.

Neste estudo, tratando-se duma análise retrospetiva, apresenta várias limitações, entre as quais se referem a omissão de dados por faltas ou abandono da consulta e a não realização de análises clínicas no período em análise. Acresce que a idade dos participantes (média 61,08 anos; faixa etária prevalente 60-69 anos), de acordo com Funnell MM et al (17), pode condicionar a assimilação e compreensão das orientações fornecidas pelos profissionais de saúde; pode associar-se a perda de motivação e/ou resistência à mudança.

No Relatório de Consenso sobre TN na pré-diabetes e diabetes (18) é reforçada a relevância da TN ministrada por um Nutricionista experiente no tratamento da diabetes, indicando, no entanto, que esta deve ser suportada por uma equipa multidisciplinar de saúde, informando e instruindo o doente diabético por forma a garantir que este faz escolhas alimentares saudáveis, de acordo com as suas necessidades, e capazes de otimizar a sua saúde. De acordo com as últimas orientações da ADA (12), os diabéticos com sobrecarga ponderal deveriam, durante 6 meses, ser acompanhados em 16 ou mais consultas multidisciplinares, garantindo deste modo uma adequada mudança do seu estilo de vida. No estudo apresentado os participantes tinham consultas com intervalo de 3 meses. Para contrariar este problema, importaria sensibilizar os órgãos ministeriais para a adequação de rácios de Nutricionistas, permitindo diminuir o intervalo de tempo entre consultas e recorrer a outras estratégias redutoras do absentismo (tais como: Ações de sensibilização e/ou atividades no âmbito da DM tipo 2, fora do horário de consulta; Motivação ao tratamento por parte de todos os profissionais de saúde envolvidos, orientando o utente para a relevância da TN na sua saúde; Reforçar, junto dos utentes, a importância de confirmação e/ou desmarcação prévia da consulta; Estabelecimento de um registo de consentimento em cada consulta, assinado pelo utente, comprometendo-se ao tratamento, entre outras). Seria também útil sensibilizar todos os profissionais para a importância do trabalho de equipa e seu papel no apoio dos utentes conducente ao cumprimento da TN.

\section{CONCLUSÕES}

O presente estudo sugere a existência de associação entre a efetividade da TN e o controlo da DM tipo 2, através de melhoria significativa de parâmetros antropométricos (peso e IMC), bem como na melhoria do controlo glicémico. Tratando-se de um estudo retrospetivo, a recolha e manutenção de dados é comprometida, pela ausência de dados suficientes, sobretudo clínicos, o que leva a que a efetividade da TN não se verifique ao longo de todo o período avaliado. Contudo, ao comparar os dados iniciais com os dados obtidos ao fim de 3 anos de seguimento em consulta de Nutrição, constataram-se melhorias significativas, particularmente nos níveis peso, IMC e glicemia em jejum, pelo que, além de indiciarem a eficácia da TN ao fim de 3 anos, estes resultados reforçam a importância da manutenção da TN individualizada a longo prazo em indivíduos com DM tipo 2. Estes resultados corroboram os de outros estudos acerca da relevância da TN, fornecida por Nutricionistas qualificados, ser eficaz e essencial no controlo da DM tipo 2. Reforçam ainda a necessidade duma monitorização periódica importante para averiguar se os objetivos da TN estão a ser atingidos, ou se existe a necessidade de estes serem reajustados. 


\section{REFERÊNCIAS BIBLIOGRÁFICAS}

1. World Health Organization. Global report on Diabetes EXECUTIVE SUMMARY. WHO. 2016; 1-4.

2. OECD. Health at a Glance 2017 OECD Indicators. 2017. OECD Publishing. 2017; $1-220$.

3. Programa Nacional para a Diabetes, Direção-Geral da Saúde. Programa Nacional para a Diabetes 2017. 2017.

4. American Diabetes Association. Facilitating Behavior Change and Well-being to Improve Health Outcomes: Standards of Medical Care in Diabetes - 2020. Diabetes Care. 2020; 43 (Suppl. 1): S48-S65.

5. Franz MJ, MacLeod J, Evert A, Brown C, Gradwell E, Handu D, Reppert A, Robinson M. Academy of Nutrition and Dietetics Nutrition practice guideline for type 1 and type 2 diabetes in adults: systematic review of evidence for medical nutrition therapy effectiveness and recommendations for integration into the nutrition care process. J Acad Nutr Diet. 2017; 117: 1659-1679.

6. Malandrucco, I., Pasqualetti, P., Giordani, et al. Very-low-calorie diet: A quick therapeutic tool to improve beta cell function in morbidly obese patients with type 2 diabetes. (2012). American Journal of Clinical Nutrition, 95(3), 609-613. Web site: https://doi.org/10.3945/ajcn.111.023697 Accessed in October 2020.

7. Kathaleen Briggs Early, Kathleen Stanley. Position of the Academy of Nutrition and Dietetics: The Role of Medical Nutrition Therapy and Registered Dietitian Nutritionists in the Prevention and Treatment of Prediabetes and Type 2 Diabetes. JOURNAL OF THE ACADEMY OF NUTRITION AND DIETETICS. 2018;118:343-353.

8. Sérgio $A$, Correia F, Breda J, et al . Programa Nacional de Combate à Obesidade. DGS. Ministério da Saúde. Circular Normativa. No: 03/DGCG. DATA: 17/03/2005.

9. Silvio E. Inzucchi, Richard M. Bergenstal, John B. Buse, Michaela Diamant, Ele Ferrannini, Michael Nauck, et al. Management of Hyperglycemia in Type 2 Diabetes, 2015: A Patient-Centered Approach Update to a Position Statement of the American Diabetes Association and the European Association for the Study of Diabetes. Diabetes Care. 2015; 38: 140-149.

10. American Diabetes Association. Obesity Management for the Treatment of Type 2 Diabetes: Standards of Medical Care in Diabetes 2020. Diabetes Care. 2020; 43 (Suppl. 1): S89-S97.

11. Diabetes Prevention Program (DPP) Research Group. The Diabetes Prevention Program (DPP): description of lifestyle intervention. Diabetes Care 2002;25:2165-2171. 12. Centers for Disease Control and Prevention. Living with Diabetes: Prevent Complications. CDC. U.S. Department of Health \& Human Services. 2019. Web site https://www.cdc.gov/diabetes/managing/problems.html?CDC_AA_ refVal=https\%3A\%2F\%2Fwww.cdc.gov\%2Fdiabetes\%2Fliving \%2Fproblems. html\#heart. Accessed in March 2019.

13. Coppell KJ, Kataoka M, Wiliams SM. Nutritional Intervention in patients with type 2 diabetes who are hyperglycaemic despite optimized drug treatment - Lifestyle Over and Above Drugs in Diabetes (LOADD) study: randomized controlled trial. BMJ. 2010; 341: 2-7.

14. Medical nutrition therapy effectiveness for specific diseases or conditions. American Dietetic Association Evidence Analysis Library. Web site. https://jandonline.org/article/ S0002-8223(10)00422-0/fulltext. Accessed in April 2019.

15. McCabe-Sellers BJ. Position of the American Dietetic Association: Integration of Medical Nutrition Therapy and Pharmacotherapy. J Am Diet Assoc. 2010; 110: 950-956.

16. Franz MJ, Boucher JL, Evert AB. Evidence-based diabetes nutrition therapy recommendations are effective: the key is individualization. Diabetes Metab Syndr Obes. 2014;7:65-72.

17. Funnell MM, Brown TL, Childs BP, et al. National Standards for diabetes selfmanagement education. Diabetes Care. 2011;34 Suppl 1(Suppl 1):S89-S96.

18. Evert AB, Dennison M, Gardner CD, Garvey WT, Lau KHK, MacLeod J, 420 et al. Nutrition Therapy for Adults With Diabetes or Prediabetes: A 421 Consensus Report. Diabetes care. 2019; 42(5):731. 